\title{
EFFECT OF ALERT PRESENTATION MODE AND HAZARD DIRECTION ON DRIVER TAKEOVER FROM AN AUTONOMOUS VEHICLE
}

\author{
Benjamin Cortens, Blair Nonnecke, Lana M. Trick \\ University of Guelph, Guelph, Ontario, Canada \\ Email: bcortens@uoguelph.ca; nonnecke@uoguelph.ca; 1trick@uoguelph.ca
}

\begin{abstract}
Summary: Autonomous vehicles are becoming increasingly common. Although the level of automation varies between vehicles even the most advanced occasionally require driver input when the driving situation is complex, or the quality of the sensory data is poor. If driver input is needed the system must alert drivers that they will have to take over but these alerts may vary in their effectiveness in prompting rapid driver takeover (time to grip the steering wheel, percentage of appropriate takeover maneuvers) and situational awareness (driver attention to the threat that necessitated take over and understanding for why take over is necessary). In this study, we used a driving simulator operating in autonomous mode to compare 2 alert types (audio-visual, and audio alone) in 3 different takeover scenarios where hazards emerged from the front (a construction zone) or the left or right side (erratic behaviour in another driver: a rogue vehicle heading toward the drivers' lane). We found that the takeover-time was faster after the audio-visual alert than the audio alert and situation awareness was better. The nature and direction of the hazard also had an effect. Situation awareness was poorer for hazards in front of the vehicle (a looming construction zone) as compared to the left and right of the driver (rogue vehicles heading toward the driver). These findings have important implications for interface design in autonomous vehicles.
\end{abstract}

\section{INTRODUCTION}

Autonomous vehicles where automated computer systems control the steering, braking, and acceleration, are becoming increasingly prevalent on the roads. However, even the most advanced autonomous vehicle occasionally requires the driver to take over because the conditions on the road exceed those for which the system was programmed (e.g., snow obscures lane markings, erratic behavior in other drivers). Consequently, autonomous systems need to rapidly signal drivers to take over driving. Furthermore, these alerting signals must provide drivers relevant information about the situation to help them understand the situation or threat that necessitated human intervention, a task that is especially challenging given that drivers may be distracted by other tasks. In this study, we compared 2 systems for signaling driver takeover, an audio-only signal and another with both audio and visual signals, assessing their relative effectiveness based on the origin and type of hazard (something that has not been explored).

If drivers are to respond appropriately to a takeover request, they need to understand the the conditions (threat or hazard) that made the takeover from automation necessary. This is critical given evidence that the situational awareness of drivers may be compromised in autonomous vehicles, impeding or delaying their response (e.g., Merat et al. 2014). At first glance, it would seem that an alerting system that signals the driver through the use of 2 sensory modalities (audition and vision) would have an advantage over one that relies on one (e.g. audition), in that 
it would give the driver more chances to perceive the alert and more information about the source of the threat (an in-vehicle display showing the origin of the threat). However, there may be disadvantages to bimodal presentation of this type. Drivers may find redundant signals annoying - and the signals themselves may serve to distract drivers from conditions on the road thus delaying response. As well, there is a danger that with redundant signals drivers might become more complacent, which means the drivers will be more "out of the loop" when required to take over. At this point, there is some support for the idea that bimodal alerts are more effective from the collision warning literature (e.g., Biondi et al. 2017), and that an audio alert can lead to increased use of visual information, (e.g., Hurwitz et al., 2010), but there are conflicting findings. For example, in a series of studies, van den Beukel (2016) found no significant differences between audio and audio-visual alerts when it came to takeover performance in an autonomous vehicle. However, the effectiveness of an alerting system may vary based on the origin of the threat and previous studies were limited to frontal hazards.

In this study, we increased the range of hazards tested, with hazards originating from the left, front and right. We compared the effectiveness of audio and audio-visual alerts in a simulated autonomous vehicle, testing drivers who were distracted by Soduko game. Takeover performance (time to grip the wheel, time to evasive maneuvers) was measured and questionnaires were used to assess situational awareness, perceived workload, as well as driver trust. We predicted that the audio-visual alerts would improve takeover performance, situational awareness and driver trust, but that the nature and position of the hazard would have an effect on the measured variables, with peripheral threats producing higher grip times, lower situational awareness, and greater workload. We also predicted that differences between audio and audio-visual alerts would be largest for peripheral (left and right) hazards as compared to front hazards.

\section{METHODS}

\section{Design and Participants}

There were 2 independent variables. The first was alert type (audio, audio-visual); participants were randomly assigned to the audio-only or the audio-visual alerts. The second was hazard position/type (left, right, or centre), a within subjects comparison with condition order counterbalanced. To ensure the alerts were put to a challenging test, we performed the study on sample of young drivers based on evidence that young drivers are both more accepting of automation in driving (Brandon \& Michael, 2014) and the least equipped to retake control (e.g., McKnight \& McKnight, 2003). Drivers 18-27 years of age were recruited from the university participant pool ( $\mathrm{n}=87,66$ females; $M$ age $=18.9$ years, $M$ years independent driving $=2.8$ ).

\section{Apparatus and Materials}

Driving Simulator and Simulations. An Oktal Driving simulator was used: a full car-body surrounded by $300^{\circ}$ wrap-around viewing screens and equipped with speakers and vibration transducers. The Oktal simulator can be switched between normal (manual) and self-driving (autonomous) mode with a toggle switch on the center console. Automation can be disengaged through manual input (steering, acceleration, braking). 
All driving simulations involved a divided highway with 3 lanes in each direction. The driver was in the center lane with vehicles in all 3 lanes ahead and behind to simulate congestion (500 vehicles/hour). The speed limit was $80 \mathrm{~km} /$ hour. Three takeover scenarios were developed to correspond to the 3 directions of interest, left, front, or right. Each scenario consisted of 4-5 minutes of autonomous driving, the hazard event ( 2.5 seconds), and 5 seconds of manual driving after the end of the hazard event. The alert occurred at the onset of the hazard (and remained on for the 2.5 second hazard duration) and the driver would have to respond within that time to avoid collision by either braking, accelerating or changing lanes. This 2.5 second duration was chosen as previous research found that 1-3 seconds was sufficient for takeover (Gold, Damböck, Lorenz, \& Bengler, 2013). The front hazard consisted of a construction zone ahead. The left and right hazards were rogue vehicles (erratic drivers) heading towards drivers from the left or right.

Automation and Alert Types. The autonomous system indicated that it was active using a blue steering wheel and cruise control indicator light. In the audio-visual condition the indicators appeared on the heads-up display (HUD), and in both audio-visual alert and audio conditions it appeared in the instrument gauge cluster (Figure 1). The blue steering wheel and cruise control indicator lights were turned off when the autonomous system was disabled.

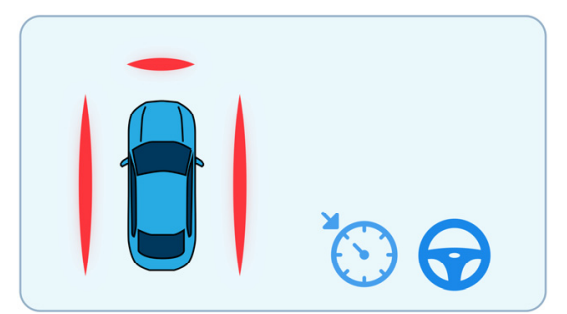

Figure 1. The heads-up-display (HUD) with takeover indicators and autonomous system state active indicators

The alerts were based on those in van den Beukel (2016). The audio alerts tones used a saw tooth (buzzing) waveform. Sounds were presented through the left, right, or front central speaker (over the driving seat) to represent left, right, or frontal hazards. The audio-visual alert combined the audio signal with a HUD: an image of the vehicle with red icons to the left, right, or front of the vehicle icon to represent the location of the hazard (see Figure 1). The HUD was chosen rather than the instrument cluster as HUDs have been shown to offer faster response to hazards compared to traditional gauge cluster presentation (Horrey \& Wickens, 2005).

Questionnaires. Situation awareness was measured using the Situation Awareness Global Assessment Technique (SAGAT) which involved direct questions about the conditions on the road (Endsley, 2000). The SAGAT measures 3 levels of situation awareness, level-1 perception, what drivers saw (e.g. vehicles and their position), level-2 comprehension, did drivers understand what they were seeing (e.g. other vehicle behaviour), and level-3 projection, were drivers able to predict what would happen next (e.g. vehicle would change lanes). Workload was assessed using the NASA-TLX (Task-Load Index), a questionnaire measuring perceived workload and performance (Hart \& Staveland, 1988). Perceived safety, helpfulness, timeliness of alerts and willingness to delegate to the autonomous system in different situations were also measured with questionnaires (21-point Likert scale where $1=$ completely disagree, $11=$ neither agree nor disagree, and $21=$ completely agree). 


\section{Procedure}

To acclimatize to the simulator drivers first did 5 minutes of hazard-free normal driving and another 5 minutes with the simulator in autonomous mode, practicing takeover 3 times. Then they did another 6-minutes in autonomous mode with no need for takeover. The 3 takeover scenarios followed. In each, participants were instructed to fill out a Sudoku puzzle while the simulator was driving in autonomous mode. They worked on this puzzle for 4-5 minutes and then there was an alert; drivers had to take control of the vehicle to avoid collision. After each scenario participants filled out the SAGAT and NASA-TLX. At the end of the study, drivers completed questionnaires on perceived safety and helpfulness of the system, the timeliness of the alerts and their willingness to delegate control to the autonomous system in different situations.

\section{RESULTS}

Most of the analyses involved fixed factorial analysis of variance (ANOVA), with GreenhouseGeisser corrections applied to the degrees of freedom in the event of violations of the sphericity assumption. Bonferroni pairwise tests were used for post-hoc comparisons of means. Partial eta squared values, $\left(\eta_{p}^{2}\right)$, were used to measure effect size. $\chi^{2}$ analyses were used to evaluate only whether evasive action taken was different between alert types and hazard directions.

As predicted, steering wheel grip response times (SWGRT), Figure 2, were significantly faster for the audio-visual than the audio-only condition $\left(F(1,61)=5.28, p=0.025, \eta_{p}^{2}=0.080, \mathrm{M}\right.$ difference $=273 \mathrm{~ms})$ and hazard direction also had a significant effect, $(F(1.91,116.41)=68.68$, $\left.p<0.001, \eta_{p}^{2}=0.530\right)$. Post-hoc tests revealed significant differences between left, front, and right hazards, with the fastest SWGRT for frontal hazards as predicted. There was a trend to smaller differences between alerts for frontal hazards but the predicted Alert X Hazard Direction interaction was not significant $\left(F(1.91,116.41)=1.65, p=0.198, \eta_{p}^{2}=0.026\right)$.
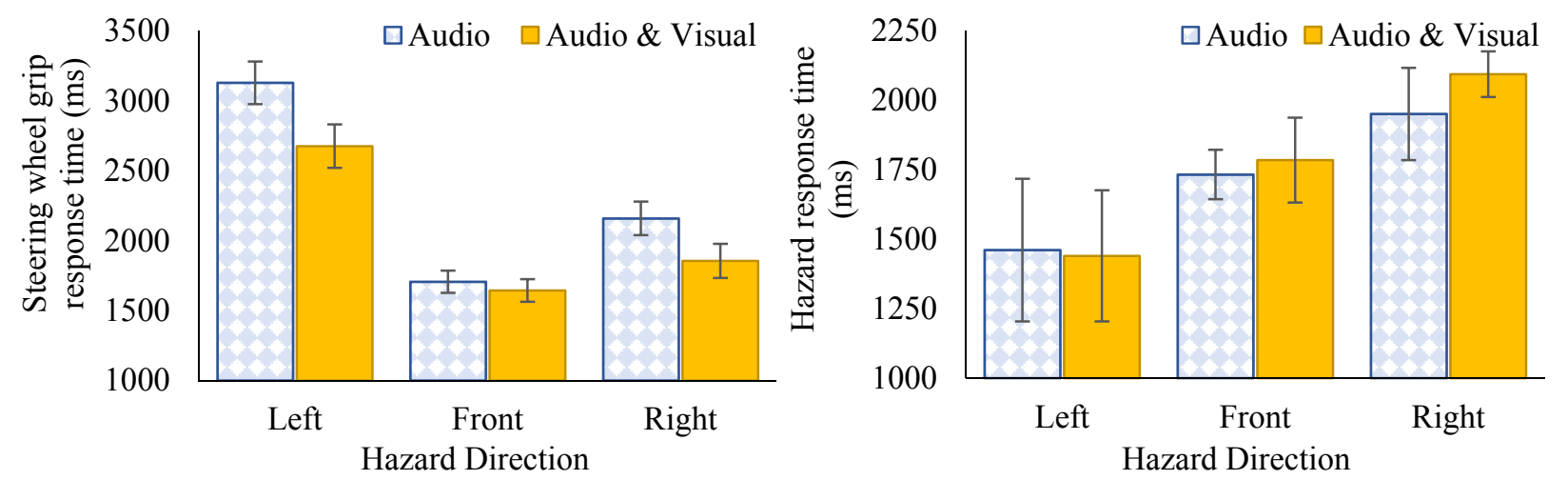

Figure 2. Steering wheel grip response time (left) and hazard response time (right) with standard error bars

Hazard response times $(H R T)$ were defined as the time between when the participant took the wheel and when they initiated their response to the hazard. There was a significant effect of hazard direction $\left(F(1.59,52.30)=5.76, p=0.009, \eta_{p}^{2}=0.149\right)$; and post-hoc tests revealed significant differences between the left and right hazards, but no significant differences between the front and either the left or the right hazards. However, contrary to prediction, neither the 
Alert or Alert X Hazard interaction were significant $\left(F(1,33)=0.14, p=0.712, \eta_{p}^{2}=0.004 ; F\right.$ $(1.59,52.30)=0.12, p=0.842, \eta_{p}^{2}=0.004$ respectively). Furthermore, alert type had no significant effect on whether or not participants took appropriate evasive action $\left(\chi^{2}(1,86)=\right.$ $0.04, p>0.100$ ) Participants took evasive action on average $80.30 \%$ of the time with audio alerts and $81.75 \%$ on average with audio-visual alerts. There was a significant effect of hazard direction $\left(\chi^{2}(2,86)=6.55, p<0.050\right)$. For frontal hazards $98.83 \%$ took evasive action, for left $79.07 \%$ took evasive action, and for right $65.11 \%$ took evasive action.

SAGAT scores can be seen from Figure 3 (higher scores indicate more situational awareness). The audio-visual alerts produced higher SAGAT scores than the audio alerts though the effect was marginal $\left(F(1,66)=3.19, p=0.079, \eta_{p}^{2}=0.046, M\right.$ difference $\left.=5 \%\right)$. Hazard direction had a significant effect though, but the pattern was opposite to that predicted, with scores for left and right hazards significantly higher than those for frontal hazards $(F(1.91,126.22)=39.99, p<$ $\left.0.001, \eta_{p}^{2}=0.377\right)$. It appears that drivers may have been more aware of the behavior of their fellow drivers than the looming construction zone in front of them. The predicted Alert X Hazard direction interaction was not significant $\left(F(1.91,126.22)=0.28, p=0.750, \eta_{p}^{2}=0.004\right)$.

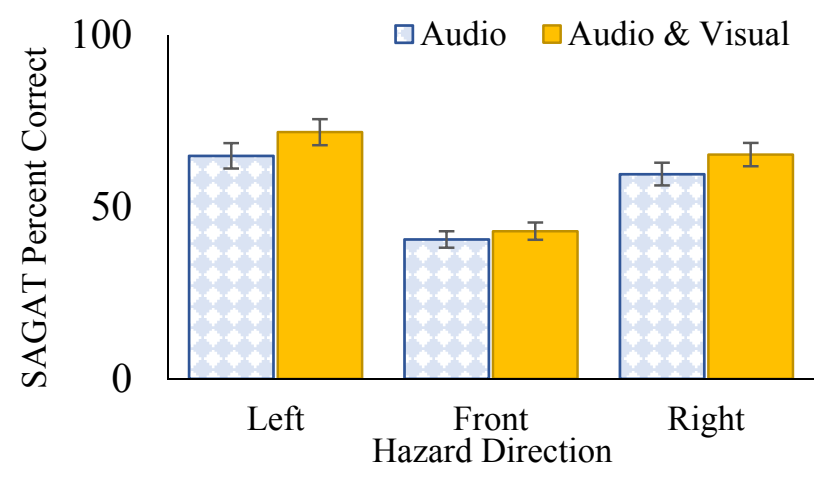

Figure 3. SAGAT total mean percent correct with standard error bars

Analysis of the NASA-TLX scores revealed significant effects for the physical workload and perceived performance scale (see Figure 4). Perceived physical workload for the audio alerts was significantly lower than that for the audio-visual alerts, which is to be expected given that there was no need to look at a display $\left(F(1,67)=4.45, p=0.039, \eta_{p}^{2}=0.062\right)$. However, perceived performance was better after the audio-visual alerts than after audio alerts $(F(1,66)=5.67, p=$ $\left.0.020, \eta_{p}^{2}=0.079\right)$. Hazard direction also had the predicted effects on perceived performance ( $F$ $\left.(1.79,118.25)=7.82, p=0.001, \eta_{p}^{2}=0.106\right)$. Post-hoc tests revealed perceived performance was significantly better for the front hazard than those in the periphery, though performance was better for the right than the left. A significant Alert X Hazard direction interaction emerged in perceived performance as well, with larger differences between directions in the audio-visual condition than the audio-only condition, $\left(F(1.79,118.253)=3.71, p=0.032, \eta_{p}^{2}=0.053\right)$.

Alert type had no significant effect on ratings of system distraction, helpfulness, safety, and whether they provided drivers with enough time to takeover $(p>0.2)$. Overall averages were 5.0, 7.5, 7.0, and 5.6 respectively based on 21 -item scales. Low values indicate that drivers did not find the systems distracting, did not perceive them to be safe or helpful; drivers felt the alerts did not give them adequate time to respond. There was also no effect of alert type on intention to 
delegate to automation ( $p>0.2)$, when over the legal limit, tired, bored, in the city, or in the country, or all the time (overall average scores: 14.8, 13.3, 9.2, 11.8, and 11.9 respectively). These averages indicate drivers were somewhat willing to delegate driving to the automated systems if they were over the legal limit for alcohol or bored (one sample $t, p<.01$ ) but were otherwise relatively neutral.
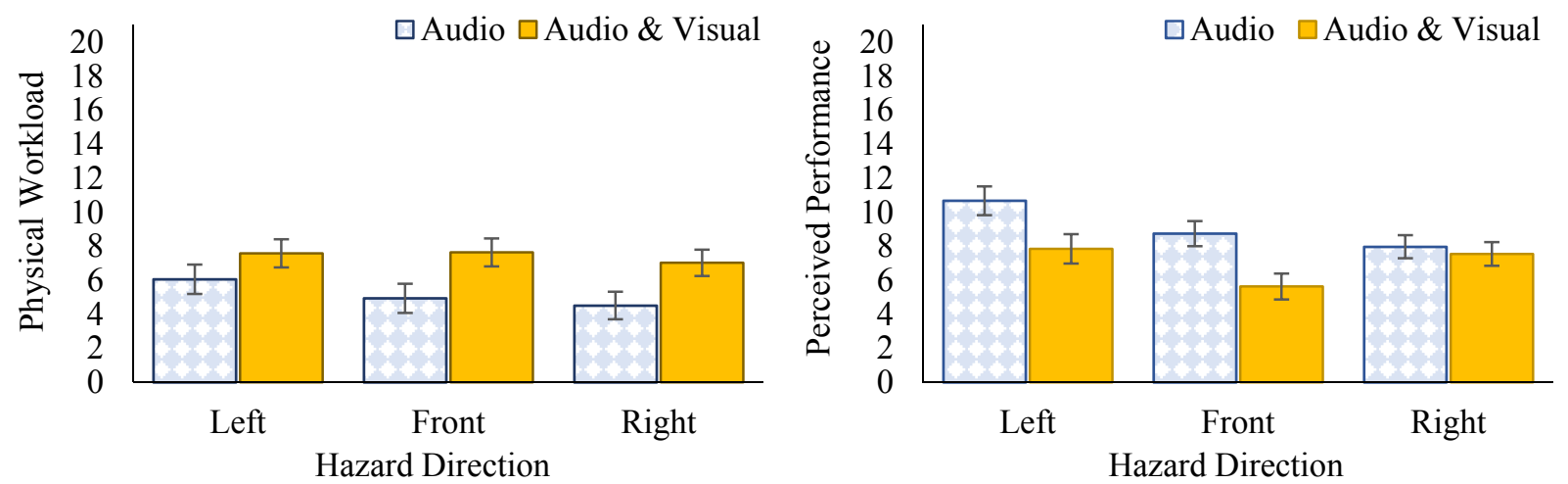

Figure 4. NASA-TLX scores with standard error bars

\section{DISCUSSION}

Although this study was only preliminary, and at this point limited to young drivers (mostly female), it provides support for the idea that in an autonomous vehicle, audio-visual takeover alerts lead to better performance than audio alone, as shown by faster SWGRT, better selfperceived performance, and higher situation awareness scores. This result is consistent with findings on collision warnings (e.g. Biondi et al., 2017) but in conflict with those on autonomous vehicle takeover (e.g., van den Beukel, 2016). Nonetheless, there were also signs of tradeoffs; HRT were slightly higher for audio-visual alerts than for audio alone and the percentage of appropriate evasive responses was slightly smaller, as might be expected if the visual displays delayed response. Neither effect was statistically significant, but these results are of concern and should be followed up in a larger scale study (more drivers, more HRT per driver). The drivers also indicated more physical workload for audio-visual than audio alerts but in many cases, the nature of the alerting cue had no effect (e.g. perceived helpfulness, safety, willingness of the driver to delegate control). In general, drivers indicated that neither system was particularly helpful or safe. Nonetheless, and alarmingly, they also indicated willingness to delegate driving to autonomous systems if they were bored or impaired (over the legal limit).

However, the limitations in this study that suggest a need for further research. First, a proper assessment of driver trust in an automated system would require more extended practice (preferably weeks). In this study, there was too little time for automation complacency to develop, and too little reason given the novelty and periodic need for takeover. Second, in assessing the benefits of alerts, it is important to measure what would have happened if there was no takeover alert. Third, although we did not have a (functional) eye tracker, eye tracking data would have improved our understanding of behaviour during takeover. We only have rough indication that participants were looking downwards (toward the Soduko game) before the alert. Finally, it is possible that relative effectiveness of the alerts vary with the nature of threat requiring takeover (Radlmayr et al. 2014). In our study, we only looked at rogue vehicle 
incursions and construction, but weather and road conditions may also necessitate takeover (e.g. disappearing lane markings, pot holes). Our research highlights the importance of alert modality design and the importance of choosing a variety of testing hazard scenarios when examining takeover safety in highly automated vehicles.

\section{ACKNOWLEDGMENTS}

This project was funded by the Natural Sciences and Engineering Council of Canada (NSERC), the Canadian Foundation for Innovation (CFI), and the Ontario Innovation Trust.

\section{REFERENCES}

Biondi, F., Strayer, D. L., Rossi, R., Gastaldi, M., \& Mulatti, C. (2017). Advanced driver assistance systems: Using multimodal redundant warnings to enhance road safety. Applied Ergonomics, 58, 238-244.

Brandon, S., \& Michael, S. (2014). a Survey of Public Opinion About Autonomous and SelfDriving Vehicles in the U.S., the U.K., and Australia. University of Michigan Transportation Research Institute, (July).

Endsley, M. R. (2000). Direct measurement of situation awareness: validity and use of SAGAT Development of Queries. Situation Awareness Analysis and Measurement, 1-21.

G.Hart, S., \& E.Staveland, L. (1988). Development of NASA-TLX (Task Load Index): Results of Empirical and Theoretical Research. Human Mental Workload, 52, 139-183.

Gold, C., Damböck, D., Lorenz, L., \& Bengler, K. (2013). “Take over!” How long does it take to get the driver back into the loop? Proceedings of the Human Factors and Ergonomics Society Annual Meeting, 57(1), 1938-1942.

Horrey, W. J., \& Wickens, C. D. (2005). Driving and Side Task Performance: The Effects of Display Clutter, Separation, and Modality. Human Factors: The Journal of the Human Factors and Ergonomics Society, 46(4), 611-624.

Hurwitz, D. S., Pradhan, A., Fisher, D. L., Knodler, M. A., Muttart, J. W., Menon, R., \& Meissner, U. (2010). Backing collisions: a study of driver's eye and backing behaviour using combined rear-view camera and sensor systems. Injury Prevention, 16(2), 79-84.

McKnight, A. J., \& McKnight, A. S. (2003). Young novice drivers: Careless or clueless? Accident Analysis and Prevention, 35(6), 921-925.

Merat, N., Jamson, A. H., Lai, F. C. H., Daly, M., \& Carsten, O. M. J. (2014). Transition to manual: Driver behaviour when resuming control from a highly automated vehicle. Transportation Research Part F: Traffic Psychology and Behaviour, 27(PB), 274-282.

Radlmayr, J., Gold, C., Lorenz, L., Farid, M., \& Bengler, K. (2014). How Traffic Situations and Non-Driving Related Tasks Affect the Take-Over Quality in Highly Automated Driving, (1988), 2063-2067.

van den Beukel, A. P. (2016). Driving automation interface design supporting drivers' changing role. Phd Thesis University of Twente. 